\title{
Approaches to Optimize and Innovate the Policies of Poverty-relief Medical Insurance Mechanism for Impoverished Population
}

\author{
—A Case Study of Hebei Province
}

\author{
Haifang FENG* \\ School of Public Administration \\ Hebei University of Economics and Business \\ Shijiazhuang, China \\ Address: 47 Xuefu Road, Shijiazhuang, Hebei \\ zip code: 050061
}

\author{
Jinguo YE \\ The Cooperative Innovation Center of the Rule of Virtue and the \\ Rule of Law in Social Management \\ Hebei University of Economics and Business \\ Shijiazhuang, China \\ Address: 47 Xuefu Road, Shijiazhuang, Hebei \\ zip code: 050061
}

\begin{abstract}
Currently, the bottleneck in the targeted poverty alleviation is the poverty due to illness or the return to poverty owing to illness. As a result, Hebei Province in August of 2016 issued Implementation Program (Trial) of Improving the Medical Assistance to Impoverished Population to Prevent Poverty Due to Illness. This article analyzes the "triple guarantee" system for the impoverished population in Hebei Province by exploring the expense-control, system design, reimbursement mechanism, service mechanism, guarantee system, and financial aid mechanism and discovers the five problems: single-channel financing, lack of guarantee for the potential impoverished population, inadequate prevention prior to illness, the incompetent role of commercial medical insurance, and the details of implementation. Guided by the spirit of 19th Party Congress-- "lengthen the short plate and strengthen the weak points", this paper proposes the approaches to optimize the medical security of the impoverished population in Hebei Province: broaden the financing channels, improve the stability and sustainability of the poverty-relief fund for medical insurance, expand the population enjoying medical security, recognize the potentially poor population and offer assistance, design competent system for the health service and disease prevention of the impoverished population, perfect the security system to bring the role of commercial insurance into full play, conduct lean management by formulating policy details so as to render the policy easily recognized.
\end{abstract}

Keywords-medical security; impoverished population; policy innovation; approaches of optimization

\section{INTRODUCTION}

One of the bottlenecks in the implementation of targeted poverty alleviation strategy is poverty due to illness. As indicated by the data on poor families established in 2013 by Poverty Alleviation Office under State Council, in China there are 12.56 million households which are thrown into poverty due to illness, $42.4 \%$ of the total. At the end of 2015 , of the registered poor households in the country, $44.1 \%$ were attributed to illness. Compared with 2013, the percentage of the poor households due to illness has substantially increased.
Illness ranks No.1 among the various reasons for poverty. To resolve the problem, Hebei Provincial Party Committee and Government in December of 2015 and August of 2016 twice published the policies for medical insurance and medical assistance, offering "triple guarantee"--basic medical insurance+critical illness insurance+medical assistance”, which have greatly improved the medical support to the impoverished people. However, by the end of 2017, Hebei Province still had 3.1 million registered poor people, of which 1,384.6 thousand were caused by illness, about $44.6 \%$ of the total, higher than the national average. Thus, how to prevent poverty due to illness is one of the challenges in alleviating poverty. As guided by the spirit of 19th Party Congress-- "lengthen the short plate and strengthen the weak points", it is necessary to optimize the approaches of alleviating poverty by assisting the impoverished population in Hebei Province so as to win the battle against poverty.

\section{INNOVATIVE POLICIES ON OFFERING MEDICAL SUPPORT} TO THE IMPOVERISHED POPULATION IN HEBEI PROVINCE

Many studies have found that medical security by cutting down the proportion of self-paid expenses can reduce the healthcare spendings and occurrence of poverty (Yardim ${ }^{[1]}$, Somkotra $^{[2]}$, Ranson ${ }^{[3]}$ ). According to Fang Yingfengand Zou Wei (2013) ${ }^{[4]}$, the rate of enjoying medical insurance by poor people or in the poor region is usually lower. The health problems aggravate the vulnerability of poverty. They hold that the public policies should encourage the investment of more resources to improve the capabilities of individuals so as to remove the vulnerability of poverty. In order to improve the poverty alleviation, Hebei Province offers "triple guarantee" service (basic medical insurance+critical illness insurance+medical assistance) and effectively eliminates poverty by providing medical support to impoverished population. 


\section{A. Innovate mechanism in controlling medical expenses and reduce the risks of healthcare expenses of impoverished population}

First of all, it is necessary to implement hierarchical medical system, improve the competency of the grass-root medical institutions, and increase the medical services so as to upgrade the medical benefits of the impoverished population. The medical services enjoyed by the poor population are negatively related to the competency of the institutions, a result of the compensation mechanism of the medical insurance. Generally, the higher the medical institution, the more competent the skills but the lower the percentage of reimbursement and the higher the base payment. The hierarchical medical system is therefore to be improved so as to narrow the difference between the payment of hospitalization costs defined by policies and the actual payment, reducing the occurrence of the accidental healthcare expenses of the poor population and the medical payment resulting in poverty. In addition, there shall be an effective supervision mechanism which conducts real-time control of medical procedures and expenses in order to regulate the services, prevent over-treatment and irrational expenses. Finally the evaluation mechanism for medical institutions has to be improved through accountability system. The assessment of the institution, the evaluation of the hospital director, performance evaluation of the director and staff shall be linked with the control of medical expenses. The weight of controlling medical expenses is to be increased in the evaluation work. The public supervision and information disclosure shall help to publicize the evaluation and inspection result from time to time and hold responsible the wrong-doers.

\section{B. Innovate the medical security system to increase the benefits of the impoverished population}

To begin with, the financial department shall provide support to the registered impoverished population, enabling all the eligible people to pay the fees. Some of the poor people due to low income find it hard to pay the premium and can't enjoy the basic medical insurance. Therefore, the provincial government decided that the financial department will pay in full amount of the premium for the registered poor people in the countryside, the members of the family with subsistence allowance, and the people with extremely difficult livelihood. With the support of basic medical needs, the impoverished population may prevent most of the risks of disastrous diseases, enjoying confidence and security. In addition, the base payment and the proportion of self-paid expenses in the medical security system has been redesigned, eliminating the "reverse subsidy" in medical resources. Thus the low-income groups can enjoy more medical resources, enhancing their benefits. For instance, the base payment for the clinical services has been eliminated, that for the hospitalization expenses of basic medical insurance have been decreased by $50 \%$, and that for the hospitalization expenses of critical illnesses has been removed. The hospitalization assistance has no base payment. The limit for the basic clinical services of medical insurance is increased to 500 Yuan/person/year, the reimbursement percentage of the basic clinical services of medical insurance is increased to $70 \%$, that of the hospitalization expenses for basic medical insurance at the county-level institutions is increased to $90 \%$, the limit of the reimbursement for hospitalization expenses of critical diseases is raised to 500 thousand Yuan/person/year. The percentage of hospitalization assistance is $80 \%$ and the limit of the assistance is 70 thousand Yuan. As a result, the hospitalization expenses will have the guarantee of "basic medical insurance+critical illness insurance+medical assistance". At the township or county hospitals, the reimbursement proportion of the impoverished population is $15 \%$ higher than that of average residents, as high as $95 \%$. At provincial and municipal tertiary hospitals, the reimbursement percentage has been increased by nearly $30 \%$, as high as $90 \%$. By the end of April 18, 2017, the 120 counties, cities and districts with registered impoverished population, had totally received clinical or in-hospital 1,153.9 thousand patients, with reimbursement at 332 million Yuan.

\section{Innovate the reimbursement of medical insurance by increasing the benefits of the impoverished population with} chronic diseases and critical illness.

As the chronic diseases and critical illness will impose too high burden on the impoverished people themselves and their family and on the other hand the current medical insurance has set up various restrictions on reimbursement. Firstly, in the basic medical insurance, the reimbursement percentage for 18 chronic diseases has been raised to $75 \%$ and the limit has been increased from 2,000 Yuan to 6,000 Yuan; that for the four critical chronic illnesses has been expanded to $90 \%$, with the reimbursement limit at 150 thousand Yuan a year. Secondly, concerning the critical diseases, the base payment has been eliminated and the reimbursement limit has been raised to 500 thousand Yuan a year. Thirdly, the medical assistance has been improved. After the reimbursement by the basic medical insurance and critical disease insurance, some families may still find it too hard to pay, returning to poverty again. The clinical expenses for critical chronic services can receive $70 \%$ subsidy, with the annual limit at 20 thousand Yuan. The assistance to hospitalization has no base payment, with the annual assistance limit at 70 Yuan, and the percentage may be as high as $80 \%$. The poor people suffering from critical illnesses, in addition to the hospitalization assistance, can receive $90 \%$ subsidy for the amount exceeding the reimbursement, with the annual limit at 200 thousand Yuan. Fourthly, as pointed out by Li Xinwei, Zhang Zhenzhong, Wang Yunping $(2007)^{[5]}$, the post-event medical assistance has to some degree impaired the effect of medical insurance against economic risks arising from diseases. By referring to the "in-event assistance" (When a patient is discharged, the hospital will deduct the reimbursement of medical insurance, exemption and medical assistance, before charging the patient. The hospital then settles the balance of the expenses with relevant departments.) In Shishou City, Hubei Province, Hebei Province has made further improvement. Since July of 2017, the county-level and lower institutions in Hebei has settled the medical expenses in the mode of "triple guarantee" . The patient with the poverty certificate and social security card or ID card needn't pay hospitalization deposit and when discharged he or she will pay for the self-paid amount. The departments for medical insurance, critical disease insurance and medical assistance will settle the account with hospitals every month. 


\section{Innovate medical insurance service mechanism to simplify the reimbursement procedures}

To begin with, Hebei integrates the social resources for medical security in the rural and urban area, coordinating the management of various resources. By 2016 the basic medical insurance system for the rural and urban residents in Hebei Province has been successfully integrated, with human resources and social security department as the administrative agencies. Moreover, the province has coordinated the cooperation between such departments as human resources and social security, finance, healthcare and family planning, civil affairs, and poverty-relief, integrating the information in various departments and setting up the information management system for medical security and assistance. By the end of September of 2017, the information system has been extended to all the medical institutions for direct settlement of medical expenses, improving the information management and service efficiency. Finally, the one-stop "triple guarantee" mode has made the service rapid and convenient. The agency handling medical insurance is to receive and examine the documents, settle the medical expenses at one spot by deducting the basic medical insurance, critical disease insurance, and medical assistance, saving the efforts of the impoverished population and offering swift services.

\section{E. Innovate the mechanism of medical insurance to meet the demand for medical assistance by impoverished population.}

In order to prevent the poverty due to illness, Hebei Province has revised the laws and regulations for the medical assistance to impoverished population. On August 18, 2016, the General Office of Hebei Provincial Department published Implementation Program (Trial) of Improving the Medical Assistance to Impoverished Population to Prevent Poverty Due to Illness (Implementation Program). The "triple guarantee" mechanism has enhanced the reimbursement for special diseases, chronic clinical diseases and hospitalization and improved the benefits of the impoverished population, reducing their burden. Subsequently, at the beginning of 2016, the five departments in Hebei--human resources and social security, civil affairs, poverty-alleviation, and finance--jointly published the Details on Implementation Program (Trial) of Improving the Medical Assistance to Impoverished Population to Prevent Poverty Due to Illness. To accelerate the application of the Implementation Program, the province published Notice of Accelerating the Improvement of Medical Assistance to Impoverished Population. To specify the benefits of the population after getting out of poverty, the General Office Department, together with poverty-relief office, civil affairs department, and financial department, published Notice on Medical Assistance to the Residents Getting out of Poverty (Ji Renshe (2017) No.108), which points out that the residents newly getting out of poverty during the consolidation period will continue to enjoy medical assistance for the impoverished population. In addition, the General Office worked with financial department and civil affairs department to publish Notice on Adjusting Fund Disbursement Mode for the Newly Added Benefits in Carrying out Implementation Program (Trial) of Improving the Medical Assistance to
Impoverished Population to Prevent Poverty Due to Illness (Ji Cai She (2017) No. 32) ), which specifies that the agency in charge of medical insurance in the human resources and social security department shall pay for the newly added fund in basic medical insurance, critical disease insurance and medical assistance for the impoverished population, and transfer the payment to the medical institutions, thus ensuring the smooth operation of the "triple guarantee". Also the General Office cooperated with healthcare and family planning commission to publish Program of Payment after Medical Services for Registered Impoverished Population in the Countryside (Ji Wei Fa (2017) No.7), which enables such people to pay no deposit for hospitalization. Finally the General Office has included all the registered impoverished population into the medical insurance for rural and urban residents, providing assistance for the self-paid amount, leaving no eligible residents beyond the medical insurance.

\section{F. Innovate the financial support to medical insurance to expand the funding sources.}

According to relevant national regulations, the added expenses for improving basic medical insurance benefits or medical security level or the assistance to the impoverished population joining medical insurance shall be not paid out of medical insurance fund. Instead, the financial department shall pay for such expenses and include them into the annual budget. The human resources and social security department in Hebei plays a leading role in coordinating with such departments as civil affairs, finance, healthcare and family planning, and poverty-alleviation to work out the measures. By the end of June of 2017, it had worked with finance department to publish the Guarantee and Disbursement Methods for Newly Added Expenses. In the second half of 2017, it worked with the financial, civil affairs, healthcare and family planning, and poverty-relief office to design the Management and Supervision of Implementation of Medical Assistance, so that the fund can be efficiently and safely utilized, ensuring the targeted poverty-alleviation campaign. At present, Hebei Province every year plans to invest 2 billion yuan to offer "triple guarantee" to impoverished population, and has defined the financial responsibilities: for the counties under the direct administration of the province, $90 \%$ the money is to be funded by the provincial department and $10 \%$ by the counties; for the rest of the counties, $80 \%$ is to be funded by the provincial department, and $10 \%$ is to be funded by the cities and counties respectively. In addition, the self-paid amount by the registered impoverished population and the special difficult population will be covered by the provincial finance and for other people calling for assistance $60 \%$ of self-paid amount will be paid by the provincial department.

\section{Major Problems Faced by Hebei Province in ENHANCING THE EFFECT OF MEDICAL SUPPORT TO IMPOVERISHED POPULATION}

\section{A. Fund for medical security: the single financing channel may generate sustained stress.}

Currently, Hebei plans to invest 2 billion Yuan every year to offer "triple guarantee" to the impoverished population. 
Take the year of 2016 as an example, when the total financial expenditure was 603.8 billion Yuan, about $0.33 \%$ being the medical support. In addition the province assigns the financial responsibilities: for the counties under the direct administration of the province, $90 \%$ the money is to be funded by the provincial department and $10 \%$ by the counties; for the rest of the counties, $80 \%$ is to be funded by the provincial department, and $10 \%$ is to be funded by the cities and counties respectively. In addition, the self-paid amount by the registered impoverished population and the special difficult population will be covered by the provincial finance and for other people calling for assistance $60 \%$ of self-paid amount will be paid by the provincial department. However, in the future there may be more and more people enjoying the medical support. Also the impoverished population and region have to pursue well-off life together with the rest of the country, calling for more funds for the medical support to the impoverished population. Of course, the government as the major party of seeking fund can ensure the stable source of fund for "triple guarantee" medical security. But the single funding channel will also bring about the potential financial pressure, restricting the financing capabilities, which in turn affect the compensation to the impoverished population. Unless some measures are taken to stimulate the enthusiasm of the various social subjects to participate in the medical support to the impoverished population and expand the funding sources, it will be rather difficult to improve further the medical assistance. In terms of the sustainability and welfare of the medical security, the current financing mechanism needs to be perfected.

\section{B. Objects of medical security: the potentially poor people} lack medical assistance, and the scope is not adequate.

In 2016 Hebei Province provided "triple guarantee" medical security to the registered impoverished population in the rural area. Chengde City started the pilot practice of offering medical assistance to other poor groups, including the people with extremely difficult livelihood, members of the families with subsistence allowances, low-income families having members with critical illness, senior citizens over 60 years old, or the only child is disabled or dead, the families unable to pay for the medical expenses or other groups with hard life. In real life, the economically poor families, the people who need long-term treatment or are disabled, the families may be thrown back into poverty due to illness, have weak resistance to disease risks. Unless a proper work is done for poverty-relief medical security, such people are more likely to join impoverished population. Therefore, the province shall expand the scope of poverty-relief medical security to include the potential impoverished population.

C. Links of medical security: Poor preventive work before illness strikes has negatively affected the poverty-relief through medical insurance.

Hebei Province has greatly increased the assistance to the chronic clinical services and the reimbursement of hospitalization expenses. The county-level and lower institutions will treat the impoverished patients before charging the fees. The "triple guarantee" settlement has lowered the threshold of the impoverished population seeing doctors. However, such assistance is still of the post-event support. Getting sick is the prerequisite for enjoying the benefits of medical insurance. Consequently, there is lack of due attention paid to the prevention of diseases, which undermines the capabilities to cope with disease-related economic risks. As a matter of fact, in order to achieve the maximum benefit of medical security, the pre-event system design is also very important. The preventive healthcare can reduce the occurrence of diseases and cut down the medical expenses, thus decreasing the probability of poverty due to illness. The 13th "five-year" plan has taken "Healthy China" as one of the national strategies, which focuses on disease prevention. Hebei Province shall follow the trend by optimizing the healthcare and medical security system.

\section{Medical security system: the commercial insurance has not been taken as a supplement.}

The "triple guarantee” medical security (basic medical insurance, critical illness insurance and medical assistance) in Hebei Province has played a leading role in getting the poor people out of poverty. However, the commercial insurance can also reduce the economic burden. At present, the commercial insurance doesn't play a competent role to diverse the risks of medical security and to help poverty-relief medical insurance. Of the multi-layer medical security, commercial insurance shall not be ignored in medical poverty-relief. So this gap shall be filled so that the commercial insurance can work together with the poverty-relief medical insurance.

\section{E. Details of medical security: The current policies fail to pay due attention to some details, affecting the public acknowledgement.}

First of all, the medical security policies for the impoverished population have distinctly improved the insurance level of chronic clinic services and hospitalization reimbursement, but fail to think of the disabled elderly in the impoverished families. In addition, for the impoverished families due to illness before the current policies were put into practice, there is no compensation program or procedures. Thirdly since July of 2017, the county-level and lower medical institutions in Hebei Province have settled the expenses in the principle of "triple guarantee". The patients with the poverty certificate and social security card or ID card needn't pay hospitalization deposit and when discharged he or she will pay for the self-paid amount. The departments for medical insurance, critical disease insurance and medical assistance will settle the account with hospitals every month. However, this policy has not been effectively explained to the impoverished population. Fourthly, in executing the policies, there is no distinction between the various low-income groups, affecting the effect of poverty-relief work. For instance, the current medical security policy for the impoverished population works much better for the low-income groups who are stuck in poverty due to critical illness than those who are poverty-stricken because of chronic diseases. In other words, the medical security for the impoverished population does not target at the specific target, resulting in the poor performance. Therefore although the current policies have generated 
substantial poverty-relief effects, there is still some work to be done before the precise poverty-relief is achieved.

\section{Approaches to Improve the Medical Insurance Assistance to IMPOVERISHed POPULATION}

A. Expand the financing channel to enhance the stability and sustainability of the fund for poverty-relief medical insurance

First of all, the government shall continue to play the leading role in financing poverty-relief medical security. The proportion of disbursement shall be about $0.3 \%$ of the public finance expenditure. While the province, city and county jointly fulfill the financial responsibility, the provincial finance department shall set aside 3-5\% fund as the adjustment fund, which will be used through transferred payment to ensure the medical security of the impoverished population in all the counties.

In addition, the public resources shall be utilized to expand the sources of the fund for poverty-relief medical security. Until now, the social resources have played a limited role in this field. On the other hand, China has abundant charitable resources. Apart from government departments, companies and public institutions, there are also more and more capable private companies, social organizations and citizens which are willing to join the poverty-relief efforts. The suggestions are: (1) set up an information platform for poverty-relief social security and a platform for medical assistance platform with the modern network and media technologies. The file of the impoverished population due to illness shall be constantly improved. The platforms will issue the information of the people calling for assistance and the needed fund. There also should be some platforms for the contributors, medical assistance, and public donation, which can collect the data on donation desire and methods of the organizations and citizens. The classified database will help to improve the social donation and expand the channel of medical assistance. (2) The human resources and civil affairs departments need to set up the foundation donated by social fund for medical support to the impoverished population, encouraging the public benefit organizations private companies and citizens to donate money to the foundation or directly to the impoverished population. The combination of the government investment and the donation of the society will help to expand the fund for poverty-relief medical assistance, improving both the efficiency and effect. (3) Perfect the stimulation mechanism to cultivate the positive atmosphere. The preferential tax deduction policies shall ensure the pre-tax deduction or exemption for the donation. The government shall regularly praise the social involvement in poverty-relief medical assistance, awarding honors to the private companies, social organizations and citizens, so as to encourage the enthusiasm of the social resources. The network, radio, TV, WeChat, newspapers and meetings are to be used to cultivate the favorable atmosphere for this campaign by reporting the good acts of the government, social organizations and citizens in participating poverty-relief medical assistance. Consequently, all walks of life will be mobilized to engage in the medical assistance to the impoverished population.

\section{B. Enlarge the scope of objects: Recognize the potentially impoverished population and offer assistance}

The objects of poverty-relief medical security have to be expanded so as to improve the effect and efficiency of the work. The author believes that as long as the basic medical services consume most or all of their fortunes, such groups shall be classified as the potential impoverished population, who should be included into the "triple guarantee" medical security for the impoverished population. But it is hard to define who potential impoverished population with an objective standard are. The first work is to recognize such people, with family budget survey, category location, regional location and community nomination. Then the potential impoverished population shall be included into the medical security assistance system through recognition and dynamic adjustment means.

Hebei has conducted some explorations in this aspect. Chengde has expanded the scope of objects and improved the standards for assistance. The low-income families are those with $150 \%$ subsistence allowances, and poverty-stricken families are those with difficult livelihood due to illness and the self-paid amount exceeds $50 \%$ of the total family income during the recent 12 months ${ }^{[6]}$. The author here proposes that it is needed to summarize the experiences in Chengde and promote the practice in the rest of the province. The standard for the potential impoverished population shall be that: the economically troubled families with income at $100-150 \%$ of the subsistence allowance, the families with a sick member who needs long-term treatment or is disabled whose average person income fails to cover the average medical expenses, or the families where critical illness results in "average family income-average family medical expenses-subsistence allowance $<0$ ". In addition, the loopholes in the subsistence allowance system shall be filled so as to ensure the fair use of social assistance fund and reduce the occurrence rate of poverty due to illness. At the same time, in some regions there may set up the limit for the self-paid amount to prevent the potential impoverished population from returning to poverty due to illness.

\section{Increase the details of the medical security: Arrange for the healthcare service and prevention for impoverished population}

The healthcare service and prevention work shall be added to the current poverty-relief medical security for the impoverished population.

To begin with, if the poor people can receive timely healthcare service, they will face much lower risks of poverty due to illness. As pointed out by 19th Party Congress, China will "improve the healthcare policies for the citizens so as to provide all-around and all-time healthcare services". However hospitals, disease control, nursing, communities and grass-root agencies have to fulfill their own duties and cooperate with each other to ensure the availability of the healthcare services to the impoverished population. There shall be a grass-root 
caretaker for the health of the poor people. At present, the healthcare services at the grass-root level are neither balanced nor competent. The poor regions and people are in an urgent demand for hierarchical treatment, contracted services of family doctors and medical alliances.

In addition, enough attention has been paid to disease prevention for the impoverished population, eliminating the hotbed for the poverty due to illness. As pointed out by 19th Party Congress, "due attention shall be paid to preventive work and it is necessary to organize patriotic health campaigns and promote the civilized and healthy lifestyle so as to control critical diseases.” In other words, there shall be some projects for public health and disease control, which are invested by the government and offered to the public free of charge. For instance, the impoverished population has access to free check-up. The three-level disease control network consisting of village clinic, township prevention and healthcare team, county disease control center shall fulfill their duties properly. Some publicity programs have to be organized to improve the consciousness of healthcare and prevention. Meanwhile it is necessary to improve the competence of grass-root medical institutions for disease prevention and treatment, and increase the services of such institutions so as to transform the purely post-event assistance to pre-event prevention plus post-event assistance. The public hospitals shall focus on the feature of "public benefits" and offer services in the poverty-stricken regions.

\section{Improve the security system to enable the commercial insurance to play a supportive role}

So far there have been some successful experiences in other provinces. For example, Shandong Province implemented the commercial insurance for medical purpose, whose annual limit shall be lower than 300 thousand Yuan ${ }^{[7]}$. The self-paid medical expenses shall be in principle no more than $10 \%$ of the total. Zhong An Insurance provides insurance products for large hospitalization expenses, helping the commercial insurance to work together with government finance and medical security fund to share the medical expenses ${ }^{[8]}$. It is advised that the medical security and civil affairs departments shall cooperate with commercial insurance institutions to add commercial insurance to the "triple guarantee", so as to form the "quadruple guarantee" assistance for medical security-- "basic medical insurance+ critical illness insurance+medical assistance+commercial insurance”.

The commercial insurance companies can rely on actuaries to calculate the premium, figure out the risks, and define the distribution of responsibilities and scope of insurance. Based on the demographic features, disease type, occurrence, medical expense and social-economic characteristics of the impoverished population, the insurance companies shall devise several good insurance products for such people. The government will then purchase such products to ensure the minimum security so that the commercial insurance can play a supportive role in the medical security of the impoverished population. The commercial insurance companies shall be encouraged to conduct information exchange, examination of medical expenses and in-time settlement of expenses for the medical security system. Some measures have to be taken to perfect the medical security system so that the resources of basic medical insurance, critical illness insurance, medical assistance, and commercial insurance can be integrated to form the cohesion in povertyrelief medical security.

\section{E. Perfect the details of medical security to perform lean management so that the policy can be widely acknowledged}

First of all, the government shall increase subsidy for medical nursing. The disabled old members in the impoverished families, where the children are too busy working to take care of them, can receive some subsidy for medical nursing when approved by the medical security agencies so as to relieve the economic burden of the family. In addition, government shall cover the medical expenses during the recent years. For the impoverished families due to illness during the past few years, the government can pay for the critical illness expenses during those years. Such families can take the documents and certificates to get some reimbursement from the critical illness insurance so that they may get out of poverty due to illness. Thirdly, some measures shall be taken to relieve the concerns of the impoverished population. The "one list" practice in Zhongxiang City, Hubei Province can be taken as reference ${ }^{[9]}$. When an object of medical assistance is hospitalized, in discharging him or her, the medical institution will offer a list of settlement covering all the deduction items, including all the details of expenses as well as the deducted amount under the "triple guarantee" concerning every item. Meanwhile, the relevant departments shall rely on WeChat, online interview and other new media to publicize the policy of medical security so as to inform the public of this policy. Finally, some steps have to be designed to distinguish the various low-income groups so as improve the precision of the policy and the poverty-relieving effect. It is necessary to find out the reason and the disease for the poverty. As for the potentially impoverished population, some money shall be provided for their training and education. For the disabled people, the key is to help them to recover. For those suffering from chronic illness and require long-term treatment, it is needed to enhance the proportion of reimbursement for clinical services so as to improve their health. For those suffering from critical illness, it is necessary to focus the assistance on hospitalization and medical assistance.

\section{CONCLUSION}

This article analyzes the "triple guarantee" system for the impoverished population in Hebei Province and discovers the five problems: single-channel financing, lack of guarantee for the potential impoverished population, inadequate prevention prior to illness, the incompetent role of commercial medical insurance, and the details of implementation. In better to achieve the goal of poverty alleviation in Hebei Province, this paper proposes the approaches to optimize the medical security of the impoverished population in Hebei Province:

broaden the financing channels to improve the stability and sustainability of the poverty-relief fund for medical insurance 
recognize the potentially poor population to expand the population enjoying medical security

design competent system for the health service and disease prevention of the impoverished population

perfect the security system to bring the role of commercial insurance into full play

conduct lean management by formulating policy details so as to render the policy easily recognized.

\section{REFERENCES}

[1] Yardim M S, Cilingiroglu N, Yardim N. Financial protection in health in Turkey: the effects of the Health Transformation Programme[J]. Health Policy \& Planning, 2014, 29(2):177-92.

[2] Somkotra T, Lagrada L P. Payments for health care and its effect on catastrophe and impoverishment: experience from the transition to Universal Coverage in Thailand[J]. Social Science \& Medicine, 2008, 67(12):2027-2035.

[3] Ranson M K. Reduction of catastrophic health care expenditures by a community-based health insurance scheme in Gujarat, India: current experiences and challenges.[J]. Bulletin of the World Health Organization, 2002, 80(8):613-621.

[4] Yingfeng Fang,Wei Zou, Capacity investment, health impact and poverty vulnerability [J]. Economic Perspectives,2013,(07):36-50. (In Chinese)

[5] Xinwei Li,Zhenzhong Zhang,Yunping Wang, et al. Investigation and analysis of urban medical assistance in Shanghai [J]. China health economics, 2007, 26 (12): 73-76. (In Chinese)

[6] Three Guarantees medical assistance will achieve one-stop reimbursement settlement[DB/OL].Hebei News Network, http://hebei.hebnews.cn/2016-09/08/content_5830236.htm.(In Chinese)

[7] Shandong province has fully implemented the medical commercial supplementary insurance for the poor [DB/OL]. http://www.moh.gov.cn/zhuz/dfxw/201705/d842c 7f0ecf5446cbb0c5ef87 2fb7b3b.shtml.(In Chinese)

[8] Should I buy web celebrity health insurance or not[DB/OL] http://news.youth.cn/gn/201704/t20170417_9501978.htm.(In Chinese)

[9] Zhixiang Wei. Research on the linkage strategy between new rural cooperative medical treatment and rural medical assistance system [D]. Northwestern university, 2011. (In Chinese) 